\title{
Bandpass Filters on a Modified Multilayer Coplanar Line
}

\author{
Atallah Balalem ${ }^{1}$, Ehab K. I. Hamad ${ }^{1}$, Jan Machac ${ }^{2}$, and Abbas S. Omar ${ }^{1}$ \\ ${ }^{1}$ Chair of Microwave and Communication Engineering, University of Magdeburg, \\ P.O. Box 4120, Magdeburg 39016, Germany \\ ${ }^{2}$ Faculty of Electrical Eng., Czech Technical University, Technicka 2, 16627 Prague 6, Czech Republic
}

\begin{abstract}
The design of compact multilayer coplanar line bandpass filters is presented. These very compact filters make use of the two sides of the substrate of the modified coplanar line. In addition, a transmission zero has been introduced to the filter response, either by extending the metalization under the ground planes, or by employing additional capacitive coupling between the different parts of the filter structure. A good agreement between the simulated and measured responses has been achieved.
\end{abstract}

Index Terms - Bandpass filter, CPW, filter, multilayer coplanar line.

\section{INTRODUCTION}

Multilayer coplanar lines (MCL) represent a potential technology for MMIC and MIC circuits [1]-[4]. The MCL was introduced as a good candidate for filter applications in [2], since it offers a wider range for a nearly constant characteristic impedance and provides more effective capacitive coupling between the resonators by locating them on the two sides of the substrate [2], [4]. Some MCL coupling structures for filters have been proposed in [3].

Many researchers have presented ways to achieve better performance of filters and make them more compact by using the originally idle area, e.g., some apertures have been opened in the ground under the narrow microstrip line to increase its inductance and in this way its characteristic impedance [5]. In [6] some apertures were opened under the coupled strips to increase the coupling factor. A modification of this is the defected ground structure (DGS), which was originally proposed for microstrip line filters [7], [8]. The DGS structure has also been introduced to CPW structures (in this case to the ground conductor located on the top substrate surface) to make the structure more compact [9], [10].

In this paper, the MCL of a modified cross section is introduced. Fig. 1a shows the cross section of the proposed MCL. Only one substrate is used and the filter is built on both sides of it. The MCL can achieve high impedance, and high coupling factors, and the resonators can be built on the rear side to enhance the coupling. This structure can be easily integrated with both CPW- and microstrip-based circuits, depending on which side of the substrate these circuits are built. This is not the case in the standard microstrip and CPW lines, as these technologies need transmission networks to be integrated together. Fig. 1b shows the cross section of the MCL, which is designed to be integrated with the standard
CPW. Fig. 1c shows the cross section of the line compatible with the microstrip line.

Three very compact bandpass filters applying the MCL structure have been designed and fabricated on an AR600 substrate of $0.787 \mathrm{~mm}$ thickness and relative dielectric permittivity of 6 . The resonators are built on the rear side of the substrate. They are coupled to the $50 \Omega$ microstrip line on the topside through the substrate, as shown in Fig. 1c. These filters are based on quasi-lumped elements [11]. The resonators of the $\mathrm{CPW}$ compatible structure, Fig. 1b, are coupled through the substrate to the strip conductor of the $50 \Omega$ CPW. In addition, a transmission zero has been introduced to the filter response by applying the capacitive coupling between the resonators.

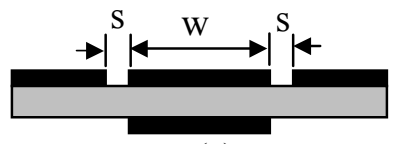

(a)

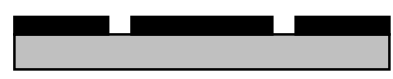

(b)

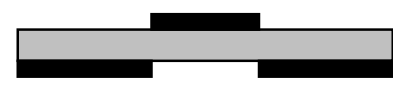

(c)

Fig. 1 Cross section of MCL (a), the MCL compatible with CPW (b), the MCL compatible with the microstrip (c).

\section{FILTER COMPATIBLE WITH THE MICROSTRIP LINE}

A bandpass filter compatible with the microstrip line has been designed. The resonators were placed on the rear side of the substrate. Fig. 2a shows the layout of the filter. The equivalent circuit model for the proposed structure is shown in Fig. 2b. Each of the two resonant circuits consists of a wide patch capacitively coupled through the substrate to the microstrip line. This coupling is provided by capacitor $\mathrm{C}_{1}$, as shown in Fig. 2b. The narrow strip mutually connecting the two patches (Fig. 2b) is represented by the series inductor $L_{1}$. This resonant structure is connected to the ground by another narrow strip represented by the shunt inductor $\mathrm{L}_{2}$ (Fig. 2b). 
Since the slot between the patch and the ground metalization is narrow, it provides a capacitive coupling between the patch and the ground, which is represented by the capacitor $\mathrm{C}_{2}$.

The structure has been simulated and optimized by the $2.5 \mathrm{D}$ electromagnetic (EM) simulator SONNET [12], and the circuit simulation has been performed on the equivalent circuit using Microwave Office [13]. Using the circuit topology shown in Fig. $2 b$ and the scattering parameters calculated by the EM simulator, the circuit parameters were tuned till a sufficient match between the circuit and the EM analyses was achieved. The resulting circuit parameters, see Fig. $2 \mathrm{~b}$, are $\mathrm{C}_{1}=0.119 \mathrm{pF}$, $\mathrm{C}_{2}=0.105 \mathrm{pF}, \mathrm{L}_{1}=2.72 \mathrm{nH}$, and $\mathrm{L}_{2}=0.407 \mathrm{nH}$. The filter was fabricated and measured. The major filter layout dimensions are stated in Fig. 2a. Fig. 3 shows the scattering parameters of the designed filter. The magnitudes of $S_{11}$ and $S_{21}$ obtained by the electromagnetic simulator, by the circuit analysis, and by measurement are plotted here. A very good agreement between the curves can be clearly seen. The rear side view of the fabricated filter is shown in Fig. 4.

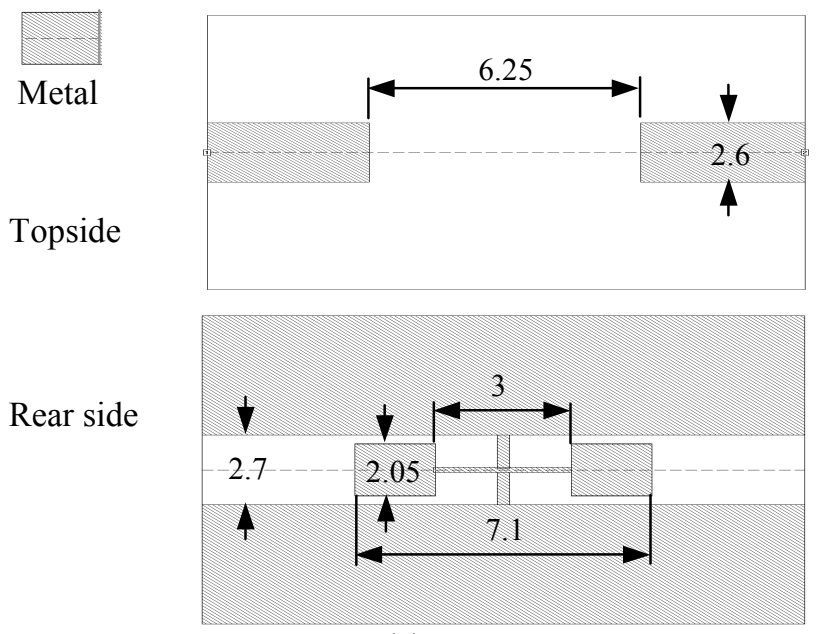

(a)

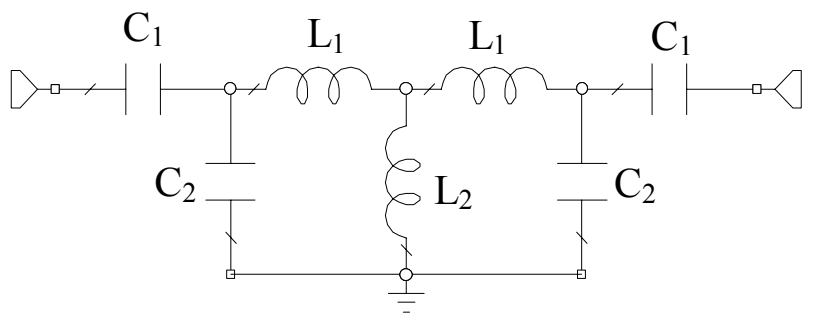

(b)

Fig. 2. Topside, and rear side layout of an MCL bandpass filter compatible with the microstrip line, Fig. 1c (a). Equivalent circuit model of the bandpass filter (b).

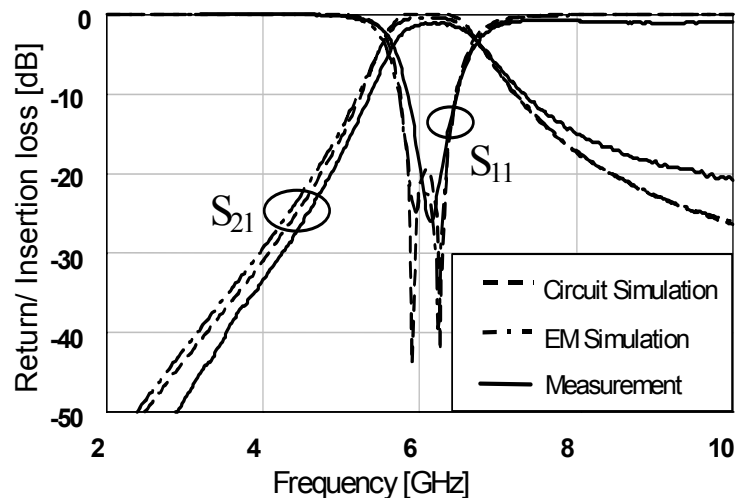

Fig. 3. Filter response, EM and circuit simulations, and the measurement.

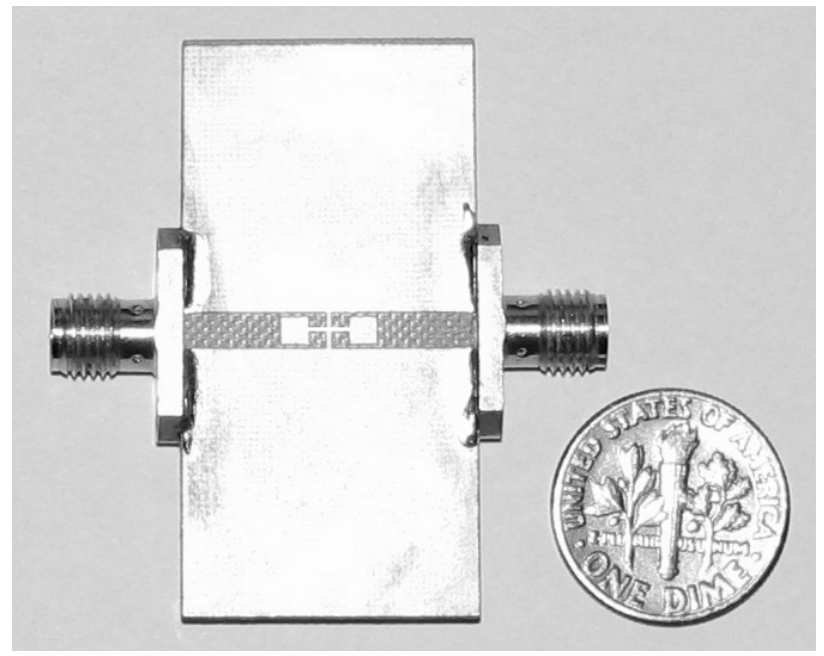

Fig. 4 The rear side view of the fabricated filter.

A transmission zero was introduced to the filter response by inserting a strong capacitive coupling between the patches. Fig. 5a shows the layout of the filter with the transmission zero, while Fig. $5 \mathrm{~b}$ shows the equivalent circuit model of this filter. The capacitive coupling between the patches is due to an additional patch located between the $50 \Omega$ lines on the topside. The slot in this patch is etched to keep the inductances of the strips on the rear side at their original value. The patches on the rear side were simultaneously widened, as is evident from a comparison of Fig. 2a with Fig. 5a. This coupling is represented in the equivalent circuit by capacitor $\mathrm{C}_{3}$. The structure was simulated and optimized using the EM simulator. The filter was fabricated and measured. Its geometrical dimensions are of the same order as those marked in Fig. 2a.

The insertion and return losses for the filter shown in Fig. 5a are demonstrated in Fig. 6. The transmission zero is introduced at about $7 \mathrm{GHz}$. The frequency shift between the simulated and measured data is caused by the discrepancies 
between the dimensions of the designed and fabricated filter layouts.

\section{BANDPASS FILTER WITH A CPW FEED LINE}

The filter with the transmission zero presented in the previous section was reconstructed. The filter is now fed by the $50-\Omega \mathrm{CPW}$, as shown in Fig. 7a. To get such a filter, the ground metalization was put on the topside instead of the rear side. The structure of the series resonators was taken from the filter shown in Fig. 2a. The shunt inductive strips are now connected to additional patches, which are capacitively coupled through the substrate to the ground. These patches are represented in the equivalent circuit of Fig. $7 \mathrm{~b}$ by capacitor $\mathrm{C}_{2}$. Since a shunt series resonator is used instead of the parallel inductance, the transmission zero is generated.

The filter was optimized by the EM simulator and then fabricated and measured. The area occupied by this filter is nearly the same as the area occupied by the filters from

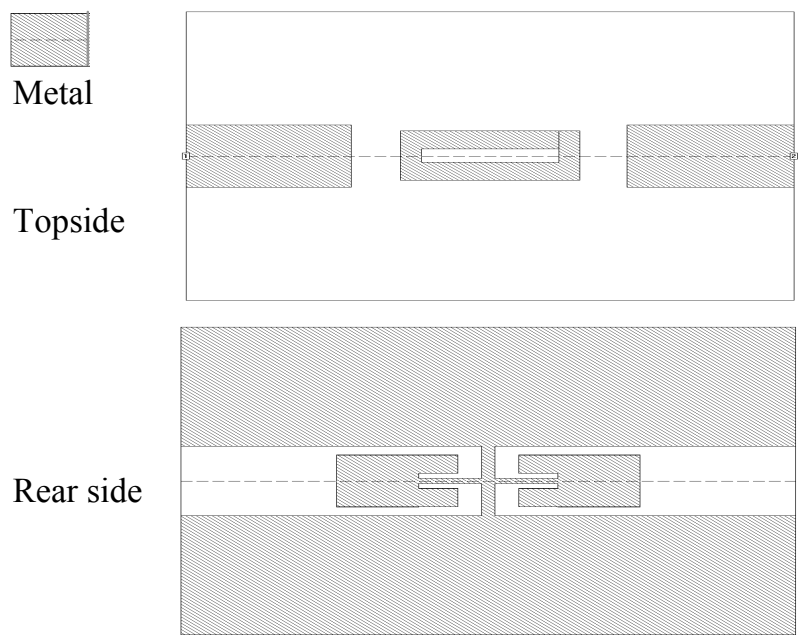

(a)

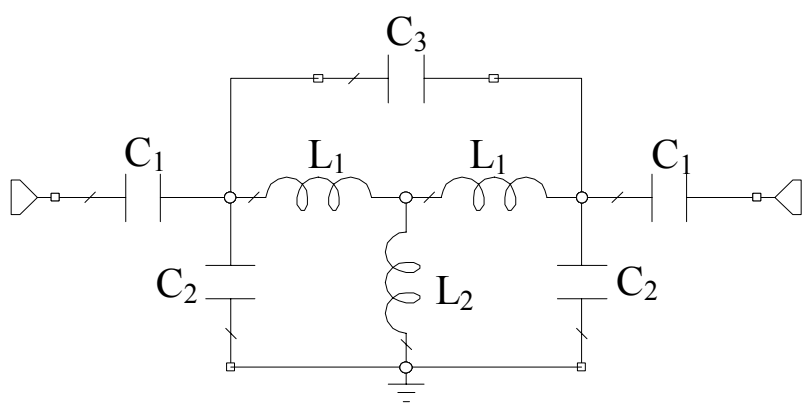

(b)

Fig. 5. Topside, and rear side layout of an MCL bandpass filter with additional capacitive coupling between the resonators (a). The equivalent circuit model (b).

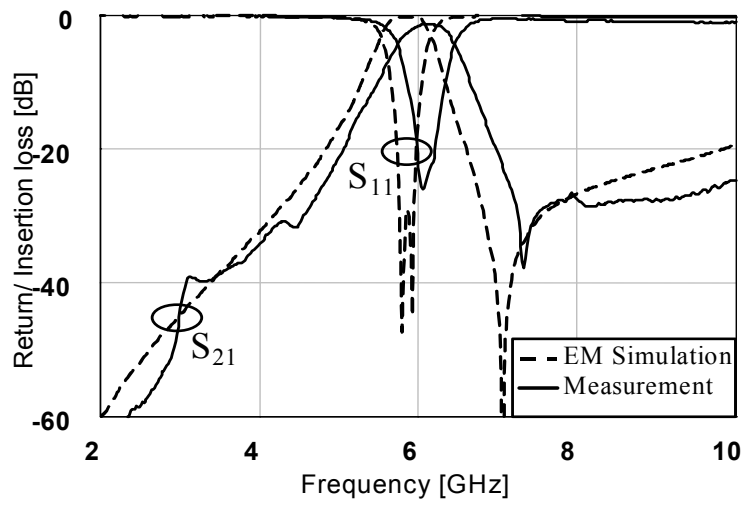

Fig. 6. The response of the filter with introduced transmission zero at $7 \mathrm{GHz}$.

Fig. 2a and Fig. 5a. The simulated filter response together with the measured amplitudes of the scattering parameters are demonstrated in Fig. 8. The frequency shift between the measured and simulated data curves is due to imperfections in the fabrication process.

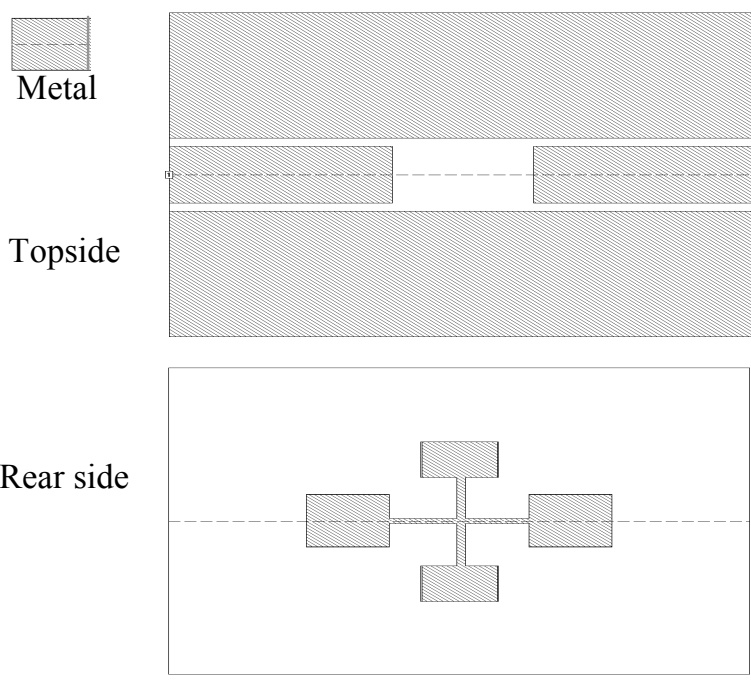

(a)

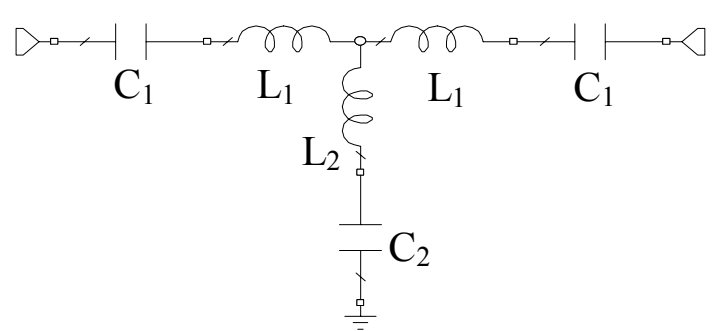

(b)

Fig. 7 Topside, and rear side layout of an MCL bandpass filter with CPW feed line (a). The equivalent circuit of the above layout (b). 


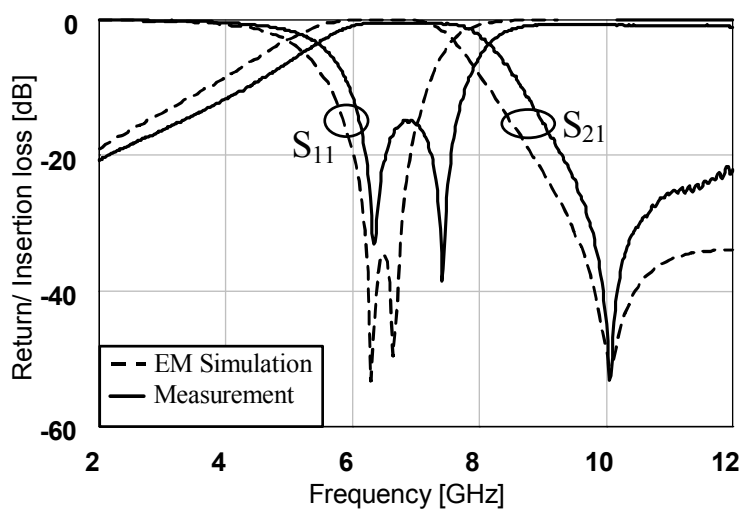

Fig. 8. Measured and simulated insertion and return loss of the bandpass filter with transmission zero of the layout shown in Fig. $7 \mathrm{a}$.

\section{CONCLUSION}

Very compact bandpass filters based on newly introduced modified multilayer coplanar lines have been presented in this paper. The main advantage of this multilayer coplanar structure is that the filter can be integrated equivalently with a CPW or a microstrip line. The behavior of these filters is simply explained by the equivalent lumped-element circuits derived from their layouts. Transmission zeros are easily added to the multilayer CPW line filter response without any major increase in its complexity or size. The filters were designed and optimized by the 2.5D EM solver to operate at a central frequency of $6 \mathrm{GHz}$. The three filters were designed and fabricated. Two of them are fed by a microstrip line and the third is fed by a CPW. The electromagnetic simulation using a commercial $2.5 \mathrm{D}$ simulator together with the circuit analysis based on the equivalent circuit give results that show a good agreement with the experiment.

\section{ACKNOWLEDGEMENT}

This research was sponsored by the Ministry of Education and Cultural Affairs in Sachsen-Anhalt, Germany. The fabrication of the filters was sponsored by the Czech Ministry of Education, Youth and Sports in the framework of the project „Research in the Area of Prospective Information and Navigation Technologies" MSM 6840770014.

\section{REFERENCES}

[1] S. Banba, T. Hasegawa, and H. Ogawa, "Multilayer MMIC branch-line hybrid using thin dielectric layers",
IEEE Microwave Guided Wave Letters, Vol. 1, pp. 346347, 1991

[2] W. Schwab, F. Boegelsack, and W. Menzel, "Multilayer suspended stripline and coplanar line filters", IEEE Transactions on Microwave Theory and Techniques, Vol. 42, No. 7, pp. 1403-1407, July 1994.

[3] W. Menzel, W. Schwab, and G. Strauss, "Investigation of coupling structure for coplanar bandpass filter", 1995 IEEE MTT-S Int. Microwave Symp. Dig., Long Beach, USA, pp.1407-1410, June 1995.

[4] C. Warns, W. Menzel, and H. Schumacher, "Transmission lines and passive elements for multilayer coplanar circuits on silicon", IEEE Transactions on Microwave Theory and Techniques, Vol. 46, No.5, May 1998.

[5] L. Zhu, H. Bu, and $\mathrm{K}$. Wu, "Unifield CAD model of microstrip line with rear side aperture for multilayer integrated circuits", 2000 IEEE MTT-S Int. Microwave Symp. Dig., Boston, USA, pp. 981-984, June 2000.

[6] L. Zhu, H. Bu, and K. Wu, "Aperture compensation technique for innovative design of ultra-broadband microstrip bandpass filter", 2000 IEEE MTT-S Int. Microwave Symp. Dig., Boston, USA pp. 315-318, June 2000.

[7] D. Ahn, J. S. Park, C. S. Kim, J. Kim, Y. Qian, and T. Itoh, "A design of the low-pass filter using the novel microstrip defected ground structure", IEEE Transactions Microwave Theory and Techniques, Vol. 49, pp. 86-93, January 2001.

[8] A. B. Abdel-Rahman, A. K. Verma, A. Boutejdar, and A. S. Omar, "Control of bandstop response of hi-lo microstrip low-pass filter using slot in ground plane", IEEE Transactions Microwave Theory and Techniques, Vol. 52, pp. 1008-1013, March 2004.

[9] M.-L. Her, Y.-Z. Wang, C.-M. Chang, and K.-Y. Lin, "Coplanar waveguide (CPW) defected ground structure (DGS) for bandpass filter application", Microwave and Optical Technology Letters, Vol. 42, No. 4, pp. 331-334, August 2004.

[10]E. K. I. Hamad, A. M. E. Safwat, and A. S. Omar, "2D Periodic Defected Ground Structure for Coplanar Waveguide", Proceedings of German Microwave Conference (GeMiC2005), Ulm, Germany, pp. 25-28, April 2005.

[11] W. Menzel, and A. Balalem, "Quasi-lumped suspended stripline filters and diplexers", IEEE Transactions Microwave Theory and Techniques, Vol. 53, No.10 pp. 3230-3237, Oct. 2005.

[12] SONNET, Version 10.52, Sonnet Software Inc.

[13] Microwave Office 2002, version 5.53, Applied Wave Research, Inc. 